\title{
Mechanisms of synchronization and pattern formation in a lattice of pulse-coupled oscillators
}

\author{
Albert Díaz-Guilera* and Conrad J. Pérez ${ }^{\dagger}$ \\ Departament de Física Fonamental, Universitat de Barcelona, Diagonal 647, E-08028 Barcelona, Spain \\ Alex Arenas \\ Departament d'Enginyeria Informàtica, Universitat Rovira i Virgili, Carretera Salou s/n, E-43006 Tarragona, Spain
}

(Received 25 September 1997)

\begin{abstract}
We analyze the physical mechanisms leading either to synchronization or to the formation of spatiotemporal patterns in a lattice model of pulse-coupled oscillators. In order to make the system tractable from a mathematical point of view we study a one-dimensional ring with unidirectional coupling. In such a situation, exact results concerning the stability of the fixed of the dynamic evolution of the lattice can be obtained. Furthermore, we show that this stability is the responsible for the different behaviors. [S1063-651X(98)11803-2]

PACS number(s): 05.90. $+\mathrm{m}, 87.10 .+\mathrm{e}, 05.50 .+\mathrm{q}$, 87.22.As
\end{abstract}

\section{INTRODUCTION}

Among the collective phenomena that are currently attracting the interest of the scientific community one of the most relevant concerns the synchronization of the temporal activity of populations of interacting nonlinear oscillators, due to its ubiquity in many different fields of science. Experimental evidences of this phenomenon have been reported for centuries [1] but in the last decades the advance in the comprehension of its nature has allowed the development of a theoretical description. In this context, several successful ideas has been suggested. An interesting approach proposed in $[2-4]$ has been shown to be useful to describe the dynamic evolution of the population. The idea consists in modeling the system as an assembly of phase oscillators interacting through continuous-time couplings. For sufficiently large coupling strength the system may undergo a phase transition from incoherence to spontaneous mutual synchronization. More challenging from a theoretical and realistic point of view is to consider networks of pulse-coupled oscillators that may account for the behavior of heart pacemaker cells, integrate and fire neurons, and other systems made of excitable units. The intrinsic nonlinearities associated with these models make their dynamical evolution more difficult to describe and only in the last years have real advances occurred [5-9].

Up to now, almost all the theoretical approaches have been centered around mean-field models or populations of just a few oscillators. From these studies it is possible to investigate the mechanisms relevant for the formation of assemblies of synchronized elements as well as other spatiotemporal structures. However, these mean-field descriptions are, in many cases, far from reality and other methods where the specific topology or geometry of the system, as well as the precise connectivity between units, must be considered because their effects may be crucial. In such a new world many points remain open. In particular the majority of works rely on simulations showing the outstanding richness

\footnotetext{
*Electronic address: albert@ffn.ub.es

${ }^{\dagger}$ Electronic address: conrad@ffn.ub.es

‡Electronic address: aarenas@etse.urv.es
}

that a low dimensional system of pulse-coupled oscillators may display. Some examples are self-organized criticality, chaos, quasiperiodicity, etc. [10].

Unfortunately, a rigorous mathematical description of these systems is still missing. Some of the theoretical papers appearing in the scientific literature prove the stability of some behaviors $[11,12]$ but they do not explore the mechanisms leading to them. The goal of this paper is twofold: the analysis of the mechanisms that are responsible for synchronization and formation of spatiotemporal structures, and, as a complement, a proof of the conditions under which they are stable solutions of the dynamical equations. Since our motivation is to analyze the essence of the problem we have considered a one-dimensional (1D) model that will allow us to illustrate the ideas in a very clear way. In spite of this apparent simplicity, this system displays a rich set of behaviors that depends on the specific values of the parameters of the model, which has been observed in lattices with higher coordination numbers $[12,13]$. Notice that populations of $1 \mathrm{D}$ pulse coupled oscillators are currently of great interest in some areas of science. As an example let us mention that for a certain type of cardiac arrhythmia there is an abnormally rapid heartbeat whose period is set by the time that an excitation takes to travel a circuit. This observation can be explained by modeling appropriately the circulation of a wave of excitation on a one-dimensional ring [14]. In a different context, synchronization and periodic states of 1D populations of phase-locked loops have been recently investigated $[11,15]$.

The structure of this paper is as follows. In Sec. II we describe the system as well as the notation used throughout the paper. Sections III and IV are devoted to analyzing the simplest cases of three and four oscillators, respectively. In Sec. V we study the general case, whereas in the last section we present our conclusions.

\section{THE MODEL}

Let us consider a system formed by a population of $N+1$ oscillators distributed on a ring. The state of each oscillator is described by its phase, which increases linearly in time, until one of them reaches a threshold value that, without loss 
of generality, we have considered equal to $\phi_{\mathrm{th}}=1$. When this happens the oscillator fires and changes the state of its rightmost neighbor according to

$$
\begin{aligned}
& \phi_{i} \geqslant 1 \Rightarrow\left\{\begin{array}{l}
\phi_{i} \rightarrow 0, \\
\phi_{i+1} \rightarrow \phi_{i+1}+\varepsilon \phi_{i+1}
\end{array}\right.=\mu \phi_{i+1}, \\
& \forall i=0, \ldots, N
\end{aligned}
$$

subjected to periodic boundary conditions, i.e., $N+1 \equiv 0$, and where $\varepsilon$ denotes the strength of the coupling. From an effective point of view, the pulse interaction between oscillators, as well as the state of each unit of the system, can be described in terms of changes in the phase, or in other words, in terms of the so-called phase response curve (PRC), $\mu \phi$ in our case. Behind this fact one assumes that the phase shift elicited by an impulse affects the period of a given unit in the current time interval but not in future intervals. In this paper we have also considered a linear PRC [16]. In practice, however, this condition can be relaxed since a nonlinear PRC does not change the qualitative behavior of the model provided the number of fixed points of the dynamics is not altered. Moreover, a linear PRC has the advantage of making the system tractable from an analytical point of view.

Let us describe the notation used in the paper. The population is ordered according to the following criterion: The oscillator that fires will be always labeled as unit 0 and the rest of the population will be ordered from this unit clockwise. After the firing, the system is driven until another oscillator reaches the threshold. Then, we relabel the units such that the oscillator at $\phi=1$ is now unit number 0 , and so on. The whole process can be described through a suitable transformation. This fact will enable us to study the origin of different structures in a very simple way. Our strategy has been to trace the phases of the oscillators after each firing and then to construct return maps either of a complete cycle, in which all the oscillators fire exactly once, or after a single firing + driving process (FD). Let us clarify this point mathematically. The first step is to construct the matrix of the transformation for a FD. To illustrate the situation let us consider the general transformation for a "jump" $n$ between two successive firings, distinguishing between $n=1$,

$$
\begin{array}{llll}
\phi_{0}=1 & \rightarrow 0 & \rightarrow 1-\mu \phi_{1} & =\phi_{N}^{\prime} \\
\phi_{1} & \rightarrow \mu \phi_{1} & \rightarrow 1 & =\phi_{0}^{\prime} \\
\phi_{2} & \rightarrow \phi_{2} & \rightarrow 1-\mu \phi_{1}+\phi_{2} & =\phi_{1}^{\prime} \\
\vdots & \vdots & \vdots & \vdots \\
\phi_{i} & \rightarrow \phi_{i} & \rightarrow 1-\mu \phi_{1}+\phi_{i} & =\phi_{i-1}^{\prime} \\
\vdots & \vdots & \vdots & \vdots \\
\phi_{N} & \rightarrow \phi_{N} & \rightarrow 1-\mu \phi_{1}+\phi_{N} & =\phi_{N-1}^{\prime}
\end{array}
$$

and $n>1$,

$\begin{array}{lcll}\phi_{0}=1 & \rightarrow 0 & \rightarrow 1-\phi_{n} & =\phi_{N-n+1}^{\prime} \\ \phi_{1} & \rightarrow \mu \phi_{1} & \rightarrow 1-\phi_{n}+\mu \phi_{1} & =\phi_{N-n+2}^{\prime} \\ \phi_{2} & \rightarrow \phi_{2} & \rightarrow 1-\phi_{n}+\phi_{2} & =\phi_{N-n+3}^{\prime} \\ \vdots & \vdots & \vdots & \vdots \\ \phi_{i} & \rightarrow \phi_{i} & \rightarrow 1-\phi_{n}+\phi_{i} & =\phi_{N-n+i+1}^{\prime} \\ \vdots & \vdots & \vdots & \vdots \\ \phi_{n-1} & \rightarrow \phi_{n-1} & \rightarrow 1-\phi_{n}+\phi_{n-1} & =\phi_{N}^{\prime} \\ \phi_{n} & \rightarrow \phi_{n} & \rightarrow 1 & =\phi_{0}^{\prime} \\ \phi_{n+1} & \rightarrow \phi_{n+1} & \rightarrow 1-\phi_{n}+\phi_{n+1} & =\phi_{1}^{\prime} \\ \vdots & \vdots & \vdots & \vdots \\ \phi_{j} & \rightarrow \phi_{j} & \rightarrow 1-\phi_{n}+\phi_{j} & =\phi_{j-n}^{\prime} \\ \vdots & \vdots & \vdots & \vdots \\ \phi_{N} & \rightarrow \phi_{N} & \rightarrow 1-\phi_{n}+\phi_{N} & =\phi_{N-n}^{\prime}\end{array}$

where $\phi^{\prime}$ describe the new phases after the FD process. The diagram describes the situation just when the leader fires (first column), the change in phases as a consequence of the emitted pulse (second), and finally the evolution of the system due to the linear driving up to the next firing (third). We believe that this is the simplest and most compact way to depict the process since we get rid of rotations that should be taken into account after the linear driving for any other relabeling method. Thus the transformation that describes this process reads

$$
\vec{\phi}^{\prime}=T_{n}(\vec{\phi}) \equiv \overrightarrow{1}+\mathrm{M}_{n} \vec{\phi}
$$

where $\vec{\phi}^{\prime}$ is a vector with $N$ components since the zeroth component does not play any role in the description. In the above expression $\mathbb{M}_{n}$ is an $N \times N$ matrix that can be written as

$$
\begin{gathered}
\left(\mathrm{M}_{1}\right)_{i j}=\delta_{i+1, j}-(1+\varepsilon) \delta_{j, 1}, \\
\left(\mathrm{M}_{n}\right)_{i j}=\delta_{i+n, j}-\delta_{j, n}+\varepsilon \delta_{j, 1} \delta_{i+n, 1} \quad \forall n>1 .
\end{gathered}
$$

In these expressions $\delta_{i, j}$ is the usual Kronecker delta. The sums should be interpreted modulus $(N+1)$ and none of the subscripts can be either 0 or $N+1$.

Since we are interested in emphasizing the mechanisms leading either to synchronization or to pattern formation, we have considered it very convenient to start our discussion with two illustrative situations where everything can be computed analytically and whose perfect understanding will help us to tackle the general case.

\section{THREE OSCILLATORS}

This is the simplest case that is worth analyzing, since the system formed by two units has been widely analyzed in the literature; see, for instance, $[6,12,17]$. If we define a simple cycle as a sequence of firings in which each oscillator fires once and only once, there are only two possibilities for this system:

(A) $0,1,2$

(B) $0,2,1$ 
provided oscillator 0 is always at the threshold at the starting point of the dynamic evolution. Here, the numbering corresponds to the firing sequence according to the initial spatial order in the lattice. Let us study both situations in detail.

\section{A. Order (A): 0,1,2}

In this case, the sequence starts when oscillator 0 fires, sending a pulse that changes the state of oscillator 1 . Afterwards, the system is driven until oscillator 1 arrives at the threshold. According to our notation this process can be viewed as

$$
\begin{aligned}
& 1 \rightarrow 0 \quad \rightarrow 1-\mu \phi_{1}=\phi_{2}^{\prime}, \\
& \phi_{1} \rightarrow \mu \phi_{1} \rightarrow 1, \\
& \phi_{2} \rightarrow \phi_{2} \rightarrow 1+\phi_{2}-\mu \phi_{1}=\phi_{1}^{\prime}
\end{aligned}
$$

Then, we have transformed a state characterized by two phases $\phi_{1}$ and $\phi_{2}$ to a new one also characterized by two phases $\phi_{1}^{\prime}$ and $\phi_{2}^{\prime}$ such that $\phi_{1}^{\prime}$ is always the phase of the oscillator that will receive the next pulse and keep this numeric order along the ring. In matrix notation the transformation can be written as follows:

$$
\left(\begin{array}{l}
\phi_{1}^{\prime} \\
\phi_{2}^{\prime}
\end{array}\right)=\left(\begin{array}{l}
1 \\
1
\end{array}\right)+\underbrace{\left(\begin{array}{cc}
-\mu & 1 \\
-\mu & 0
\end{array}\right)}_{\mathbb{M}_{1}}\left(\begin{array}{l}
\phi_{1} \\
\phi_{2}
\end{array}\right)
$$

The complete cycle is constructed by applying three times this transformation $\left[T_{1} \circ T_{1}^{\circ} T_{1}(\phi)\right]$. In other words,

$$
\vec{\phi}^{\prime \prime \prime}=\vec{R}_{A}+\mathbb{M}_{A} \cdot \vec{\phi}
$$

The independent term $\vec{R}_{A}$ is

$$
\vec{R}_{A}=\left(\overrightarrow{\overrightarrow{1}}+\mathrm{M}_{1}+\mathrm{M}_{1} \cdot \mathrm{M}_{1}\right) \cdot \overrightarrow{1}
$$

where $\overrightarrow{1}$ is a column vector of 1 's, $\overrightarrow{1}$ is the identity matrix, and the matrix of the transformation $\mathrm{M}_{A}$ is defined as

$$
\mathrm{M}_{A}=\mathrm{M}_{1} \cdot \mathrm{M}_{1} \cdot \mathrm{M}_{1}
$$

From this expression it is easy to compute the fixed points of the transformation, which are solutions of the equation

$$
\vec{\phi}^{*}=\vec{R}_{A}+\mathbb{M}_{A} \cdot \vec{\phi}^{*},
$$

that is,

$$
\begin{aligned}
& \phi_{1}^{*}=\frac{2}{3+2 \varepsilon} \\
& \phi_{2}^{*}=\frac{1}{3+2 \varepsilon} .
\end{aligned}
$$

The stability of these fixed points is given by the eigenvalues of $\mathbb{M}_{A}$, which are

$$
\frac{-(\mu-3) \mu^{2} \pm i(\mu-1) \mu^{3 / 2} \sqrt{4-\mu}}{2}
$$

whose moduli are $\mu^{3 / 2}$. Depending on the sign of $\varepsilon$ the fixed point is either stable $(-)$ or unstable $(+)$.

\section{B. Order (B): 0,2,1}

Now, oscillator 1 receives the pulse but it is oscillator 2 that leads the driving and arrives first to the threshold

$$
\begin{aligned}
& 1 \rightarrow 0 \quad \rightarrow 1-\phi_{2}, \\
& \phi_{1} \rightarrow \mu \phi_{1} \rightarrow 1+\mu \phi_{1}-\phi_{2}, \\
& \phi_{2} \rightarrow \phi_{2} \rightarrow 1 .
\end{aligned}
$$

Therefore, the new phases are

$$
\left(\begin{array}{l}
\phi_{1}^{\prime} \\
\phi_{2}^{\prime}
\end{array}\right)=\left(\begin{array}{l}
1 \\
1
\end{array}\right)+\underbrace{\left(\begin{array}{ll}
0 & -1 \\
\mu & -1
\end{array}\right)}_{\mathbb{M}_{2}}\left(\begin{array}{l}
\phi_{1} \\
\phi_{2}
\end{array}\right)
$$

Again the complete cycle is constructed by applying three times this transformation $\left[T_{2} \circ T_{2} \circ T_{2}(\phi)\right]$ :

$$
\vec{\phi}^{\prime \prime \prime}=\vec{R}_{B}+\mathbb{M}_{B} \cdot \vec{\phi}
$$

where the independent term $\vec{R}_{B}$ is now

$$
\vec{R}_{B}=\left(\overrightarrow{\overrightarrow{1}}+\mathbb{M}_{2}+\mathbb{M}_{2} \cdot \mathbb{M}_{2}\right) \cdot \overrightarrow{1}
$$

and the matrix of the transformation is

$$
\mathrm{M}_{B}=\mathrm{M}_{2} \cdot \mathrm{M}_{2} \cdot \mathrm{M}_{2}
$$

The fixed point of this transformation, which is the solution of the equation

$$
\vec{\phi}^{*}=\vec{R}_{B}+\mathrm{M}_{B} \cdot \vec{\phi}^{*},
$$

is

$$
\begin{aligned}
& \phi_{1}^{*}=\frac{1}{3+\varepsilon}, \\
& \phi_{2}^{*}=\frac{2+\varepsilon}{3+\varepsilon} .
\end{aligned}
$$

The stability of this fixed point is given by the eigenvalues of $\mathrm{M}_{B}$ that are

$$
\frac{3 \mu-1 \pm i(\mu-1) \sqrt{4 \mu-1}}{2},
$$

whose moduli are again $\mu^{3 / 2}$. Therefore, both fixed points describe the same physical behavior that is independent on the particular order in which oscillators fire. 


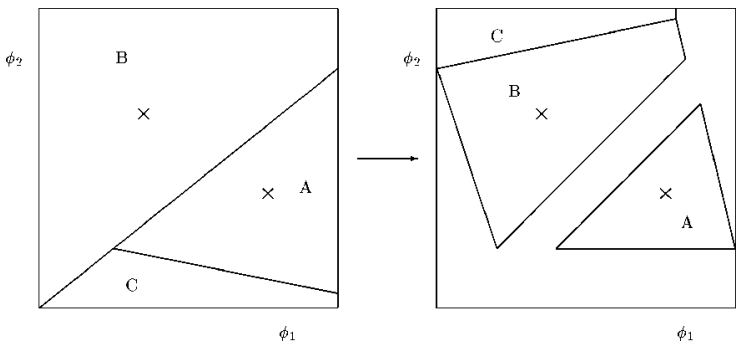

FIG. 1. Evolution of the phases $\phi_{1}$ and $\phi_{2}$, for a negative value of $\varepsilon$. Right: initial configurations when oscillator 0 is at the threshold value; left: new values of the phases when oscillator 0 reaches the threshold again. The crosses correspond to the fixed points and each region corresponds to a given sequence of firings (see text).

\section{Phase ordering}

In the previous subsections we have studied some features of the final state of the system when a sequence of identical transformations are applied successively. However, the reader can argue that those sequences are not the only possible dynamical evolution. Indeed, to deal with all the possible situations we should analyze what happens when a mixture of $T_{1}$ and $T_{2}$ are combined in an arbitrary manner to complete a cycle, and what sort of physical consequences derive from this fact. In addition, one may wonder whether an advancement can take place, i.e., if a given oscillator can fire twice before another element of the chain arrives at the threshold, breaking thus our definition of a simple cycle. Such issues are discussed in this subsection. To illustrate this point let us start by considering Fig. 1, where we have plotted the evolution of the phases each time oscillator 0 is at the threshold value. It is obvious that there are two different situations: a positive or negative value of the coupling. In this figure we have analyzed the situation for $\varepsilon<0$.

The left hand side of Fig. 1 shows all the possible initial configurations and the right hand side shows how they transform when oscillator 0 is again at the threshold. Regions (A) and $(\mathrm{B})$ represent the sequences $(\mathrm{A})$ and $(\mathrm{B})$ described before, respectively. Here, we can see that states lying initially in one of these regions will approach the fixed points (attractors), since (A) and (B) are slowly shrinking. Therefore, once one starts with a given sequence no other one can be applied to describe the dynamical evolution of the system. The physical picture associated to the attractor fixed point is quite simple. The oscillators remain at a certain distance in the phase space (phase locking). For larger dimensions (more oscillators) this fact induces the creation of complex spatial patterns.

Special attention is given to region (C), which corresponds to a sequence of firings 010 . This means that oscillator 0 advances to oscillator 2, a situation not covered before. Here we can see that the effect of this advancement is to reorganize the phases in such a way that after one cycle the old configurations fall in the basin of attraction of region (B) and therefore, for them, sequence (B) must be applied forever. No more advancements can take place. The main conclusion is that advancements play a role only in the transient but not in the stationary properties of the dynamics. A more clear picture of the physical meaning of this fact will be provided in the next section.

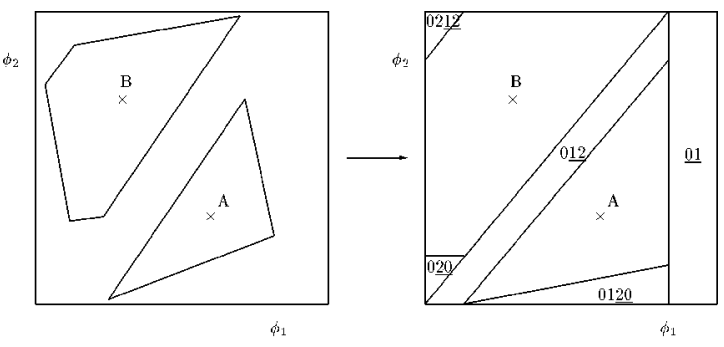

FIG. 2. The same as Fig. 1 for positive coupling.

The mechanism for positive $\varepsilon$ is the opposite of that for negative $\varepsilon$. Regions (A) and (B) are enlarged every cycle, and the configurations move away from the fixed points (repellers) until they cross some of the borders where at least two oscillators get absorbed and synchronize. Figure 2 shows this fact. The left hand side of the figure depicts the size of the basin of attraction of those configurations (closed lines) that when oscillator 0 reaches the threshold again still require sequence (A) or (B) to evolve dynamically. The rest of the phase space is formed by states characterized by the fact that after the next firing of oscillator 0 occurs at least two units will merge. These units are specified (underlined) on the right hand side of the figure. Since two synchronized oscillators act as a single one and cannot be broken after a complete cycle, the problem is now equivalent to that of two oscillators. This dimensional reduction is the essence of synchronization. For mean-field models the word absorption has been used to illustrate this phenomenon [6].

To address the question about the plausibility of having mixtures of consecutive transformations $T_{1}$ and $T_{2}$ it is convenient to look at the problem from another perspective. Instead of considering complete cycles it is better to analyze single firings. Let us suppose for simplicity negative (inhibitory) coupling between units. In this case, it is evident that the alternative application of both transformations leads to two possible options. The first case to be considered is the combination $T_{1} \circ T_{2}$. We can observe that applying $T_{2}$ is inconsistent with the application of $T_{1}$ afterwards, because the resulting configuration $T_{2}(\vec{\phi})$ does not satisfy the possible different phase orders necessary to apply $T_{1}$. On the other hand, the order $T_{2} \circ T_{1}$ implies one advancement between oscillators, corresponding to region (C) (see Fig. 1) of phase space. This situation has been discussed previously to play the role of transient dynamic behavior of the system. Thus we can conclude that, in general, the advancements will cause the phases to be reordered until the system reaches a configuration that is consistent with only one sequence of transformations.

\section{FOUR OSCILLATORS}

In this case there are 6 different orders for the oscillators to complete a simple cycle:

(A) $0,1,2,3$

(B) $0,1,3,2$

(C) $0,2,1,3$

(D) $0,2,3,1$

(E) $0,3,1,2$

(F) $0,3,2,1$

Analogously to the three oscillators case we define the 
matrices of the transformation between successive firings of two oscillators, according to the jump between oscillators that fire successively:

$$
\begin{aligned}
& \mathbb{M}_{1}=\left(\begin{array}{ccc}
-\mu & 1 & 0 \\
-\mu & 0 & 1 \\
-\mu & 0 & 0
\end{array}\right), \\
& M_{2}=\left(\begin{array}{rrr}
0 & -1 & 1 \\
0 & -1 & 0 \\
\mu & -1 & 0
\end{array}\right), \\
& \mathbb{M}_{3}=\left(\begin{array}{lll}
0 & 0 & -1 \\
\mu & 0 & -1 \\
0 & 1 & -1
\end{array}\right) .
\end{aligned}
$$

We can easily compute the eigenvalues of those matrices. For $M_{1}$ and $M_{3}$ the eigenvalues have moduli larger (smaller) than 1 for positive $\varepsilon$ (negative $\varepsilon$ ). However, for $M_{2}$ there is one eigenvalue with modulus equal to 1 . This will be very important when discussing the stability of the fixed points.

According to these jumps the transformation of a complete cycle for the different orders are constructed in the following ways:

(A) $0,1,2,3 \rightarrow T_{1} \circ T_{1} \circ T_{1} \circ T_{1}$,

(B) $0,1,3,2 \rightarrow T_{2} \circ T_{3} \circ T_{2} \circ T_{1}$,

(C) $0,2,1,3 \rightarrow T_{1} \circ T_{2} \circ T_{3} \circ T_{2}$,

(D) $0,2,3,1 \rightarrow T_{3} \circ T_{2} \circ T_{1} \circ T_{2}$

(E) $0,3,1,2 \rightarrow T_{2} \circ T_{1} \circ T_{2} \circ T_{3}$,

(F) $0,3,2,1 \rightarrow T_{3} \circ T_{3} \circ T_{3} \circ T_{3}$.

For instance, let us write, in matrix form, the transformation for the case $(\mathrm{B})$ :

$$
\begin{aligned}
\vec{\phi}^{\prime}= & \overrightarrow{1}+\mathbb{M}_{2} \cdot \overrightarrow{1}+\mathbb{M}_{2} \cdot \mathbb{M}_{3} \cdot \overrightarrow{1}+\mathbb{M}_{2} \cdot \mathbb{M}_{3} \cdot \mathbb{M}_{2} \cdot \overrightarrow{1} \\
& +\mathbb{M}_{2} \cdot \mathbb{M}_{3} \cdot \mathbb{M}_{2} \cdot \mathbb{M}_{1} \cdot \vec{\phi}
\end{aligned}
$$

We can now proceed to compute the fixed points associated to these transformations which are:

$$
\begin{aligned}
& \text { (A) } 1, \quad \frac{3}{4+3 \varepsilon}, \quad \frac{2}{4+3 \varepsilon}, \frac{1}{4+3 \varepsilon} . \\
& \text { (B) } 1, \quad \frac{1}{2+\varepsilon}, \quad 0, \frac{1+\varepsilon}{2+\varepsilon} . \\
& \text { (C) } 1, \quad \frac{1}{2+\varepsilon}, \quad 1, \frac{1}{2+\varepsilon} . \\
& \text { (D) } 1, \quad \frac{1}{2+\varepsilon}, \quad 1, \frac{1}{2+\varepsilon} . \\
& \text { (E) } 1, \quad \frac{1}{2+\varepsilon}, \quad 0, \frac{1+\varepsilon}{2+\varepsilon} . \\
& \text { (F) } 1, \quad \frac{1}{4+\varepsilon}, \quad \frac{2+\varepsilon}{4+\varepsilon}, \frac{3+\varepsilon}{4+\varepsilon} .
\end{aligned}
$$

Numerically, these fixed points are unique since this is ensured by the fact that all the eigenvalues of the matrix that multiply $\vec{\phi}$ are different from 1 in all cases. Nevertheless the fixed points for cases (C) and (D) are senseless since they do not verify the prescribed order, and hence they are physically unacceptable. Furthermore, the fixed points of cases (B) and (C) are physically the same since they just differ in the order in which oscillators 0 and 2 fire but they are synchronized. Thus, basically, we have to deal with fixed points where the phase difference between adjacent oscillators is roughly $\phi_{i+1}-\phi_{i}=1 / 4,2 / 4,3 / 4(\bmod 1)$, with small corrections depending on $\varepsilon$.

Let us focus now on the process from the point of view of single firings again. Notice that $T_{1}$ and $T_{3}$ are transformations that can be applied alone, successively leading to a natural complete cycle. However, $T_{2}$ cannot be applied in this way because this would lead to an unphysical situation where only a group of oscillators will fire. This means that $T_{2}$ must be combined with $T_{1}$ or $T_{3}$ in order to be physically acceptable; then the effect of having an eigenvalue with modulus 1 , that can carry a metastability on the system, is avoided by this combination. The combinations of $T_{2}$ with either $T_{1}$ or $T_{3}$, can be done in the way described by the orders (B), (C), (D) or (E), that always give rise to a chessboard-type pattern. Other combinations can be obtained applying $T_{1} \circ T_{2}$ or $T_{3} \circ T_{2}$ forever; this situation elicits advancements but nevertheless the resulting patterns are chessboard type again.

\section{V. $N+1$ OSCILLATORS}

Although, in general, to construct a complete cycle is not a trivial mechanism we can infer some key points about the behavior of the system from the FD processes as we have done for the simplest lattices: compute the fixed points and their stability.

\section{A. Fixed points}

Due to their different behavior we will have to distinguish again the cases $n=1$ and $n>1$. Thus, for the first situation, we have to solve

$$
\begin{gathered}
\phi_{1}=1-\mu \phi_{1}+\phi_{2}, \\
\phi_{2}=1-\mu \phi_{1}+\phi_{3}, \\
\vdots \\
\phi_{N-1}=1-\mu \phi_{1}+\phi_{N}, \\
\phi_{N}=1-\mu \phi_{1} .
\end{gathered}
$$

Simply summing up all the equations we are left with $\phi_{1}=N\left(1-\mu \phi_{1}\right)$ and then we get

$$
\phi_{1}^{*}=\frac{N}{N+1+N \varepsilon} .
$$

Notice that the other phases at the fixed point can be obtained from this one, since $\phi_{j-1}-\phi_{j}=1-\mu \phi_{1} \forall j$ $=2, \ldots, N$. This fixed point corresponds to a situation in which all the oscillators fire in turn following their lattice ordering. 
On the other hand, for $n>1$ we have to solve

$$
\begin{gathered}
\phi_{1}=1-\phi_{n}+\phi_{n+1}, \\
\vdots \\
\phi_{N-n}=1-\phi_{n}+\phi_{N}, \\
\phi_{N-n+1}=1-\phi_{n}, \\
\phi_{N-n+2}=1-\phi_{n}+\mu \phi_{1}, \\
\phi_{N-n+3}=1-\phi_{n}+\phi_{2}, \\
\vdots \\
\phi_{N}=1-\phi_{n}+\phi_{n-1} .
\end{gathered}
$$

Summing up again all the equations we now obtain

$$
\phi_{1}+\phi_{n}=N\left(1-\phi_{n}\right)+\mu \phi_{1} \text {. }
$$

Now we notice that it is not enough to get one of the values of the phases. We have to close the system of equation by means of the following procedure:

$$
\phi_{1}=1-\phi_{n}+\underbrace{\phi_{n+1}}_{1-\phi_{n}+\phi_{2 n+1}}=2\left(1-\phi_{n}\right)+\phi_{2 n+1}
$$

and so on. Again all the subscripts are understood modulus $N+1$. This procedure is repeated until we reach $\phi_{N-n+1}$, which closes the dependence of $\phi_{1}$ on $\phi_{n}$. Obviously, a necessary condition to close it is that $N+1$ and $n$ do not have common factors. This procedure is iterated $m_{n}-1$ times, where $m_{n}$ verifies

$$
\left(1+m_{n} n\right) \bmod (N+1)=0,
$$

and it exists and is unique for each $n<N+1$. Then for a given $N$ we will have to consider all the values of $m_{n}$ between 1 and $N$ without common factors. We can therefore obtain that at the fixed point,

$$
\phi_{n}^{*}=\frac{N+m_{n} \varepsilon}{N+1+m_{n} \varepsilon}, \quad \phi_{1}^{*}=\frac{m_{n}}{N+1+m_{n} \varepsilon} .
$$

Again, this situation would correspond to a sequence of FD processes of jump $n$. It is easy to convince oneself that in both cases $(n=1$ and $n>1)$ one can build a complete cycle by applying $N+1$ times the transformation $T_{n}$, which does not change the fixed points. In principle, this successive application could make new fixed points appear, but this is forbidden by the fact that the moduli of the eigenvalues of $\mathrm{M}_{n}$ are always larger than 1 for $\varepsilon>0$, and smaller than 1 in the opposite case, whenever $N+1$ and $n$ do not have common factors, as we show in the Appendix.

But we still do not know what happens when $N+1$ and $n$ have common factors. As we show in the Appendix in this case there exists at least one eigenvalue of modulus 1 and this fact does not ensure the existence of a solution for the set of algebraic equations; even when this solution can exist it usually gives rise to unphysical situations, as, for instance, to values of the phases that are either below zero or above one. We showed explicitly for the case of 4 oscillators that under these circumstances the transformation of an FD process of this kind has to be combined with other transformations with noncommon factors and that this led to advancement between oscillators and to the formation of a chessboardlike pattern. This is also what happens in the general case. The matrix associated with this combination will have eigenvalues with moduli different from 1 and will guarantee the existence of the fixed point. Let us assume that a given spatial structure with period $p=(N+1) / n$ exists and then there are some oscillators that are synchronized $\left(\phi_{0}=\phi_{p}=\phi_{2 p}\right.$ $\left.=\cdots=\phi_{(n-1) p} ; \phi_{1}=\phi_{p+1}=\ldots ; \ldots\right)$. Hence we need only to consider each spatial period, since the transformations of jump $p$ correspond to oscillators that fire exactly at the same time. Within each period there will be different possibilities for the magnitude of the jumps $n_{p}$ and then the fixed points will be characterized by

$$
\phi_{1}^{*}=\frac{m_{n_{p}}}{p+m_{n_{p}} \varepsilon}=\frac{n m_{n_{p}}}{N+1+n m_{n_{p}} \varepsilon} .
$$

By combining Eqs. (27), (30), and (31), we realize that for a given $N+1$ there always exists a value of $m$ $(0<m<N+1)$ such that

$$
\phi_{1}^{*}=\frac{m}{N+1+m \varepsilon}
$$

will be the phase of oscillator 1 at a fixed point. We have used this fact to identify the fixed points of the dynamics in simulations of lattices of a few oscillators, as we will see later on.

In principle one can still think about the possibility of other fixed points corresponding to combinations of transformations not described above, for instance, successive applications of two transformations of different values of $n$; but this case will necessarily involve advancements between the oscillators, which, as we have already discussed, are only important in the transient but not in the approach to the final state. This, of course, can make the transients become quite large, as we have observed in the computer simulations, but the only final states for an inhibitory coupling are those described earlier in the text. It is also important to note that depending on the strength of the coupling, and on the number of oscillators, there will be some fixed points, for an inhibitory coupling, that will not exist, i.e., those that verify

$$
\varepsilon<1-\frac{N+1}{m}
$$

For instance, for the three oscillators case this happens for $m=2$ when $\varepsilon<-0.5$ [see Eq. (10)] and then region (A) disappears and the fixed point corresponding to $(\mathrm{B})$ is the only possible final state. 
TABLE I. Percentage of the final states the system formed by $N+1$ oscillators reaches, for $\varepsilon=-0.1$. The first column stands for $N+1$ and the first row for $m$ in Eq. (32), which approximately corresponds to the phase difference times $N+1$ between consecutive oscillators. It is averaged for 1000 initial random configurations picking each phase from a uniform distribution between 0 and 1 .

\begin{tabular}{|c|c|c|c|c|c|c|c|c|c|c|}
\hline & 1 & 2 & 3 & 4 & 5 & 6 & 7 & 8 & 9 & 10 \\
\hline 3 & 61.4 & 38.6 & & & & & & & & \\
\hline 4 & 21.2 & 71.3 & 7.5 & & & & & & & \\
\hline 5 & 5.5 & 56.5 & 37.2 & 0.8 & & & & & & \\
\hline 6 & 1.2 & 29.1 & 58.0 & 11.7 & 0.0 & & & & & \\
\hline 7 & 0.0 & 11.3 & 51.7 & 34.2 & 2.5 & 0.0 & & & & \\
\hline 8 & 0.0 & 3.8 & 32.1 & 50.6 & 13.2 & 0.4 & 0.0 & & & \\
\hline 9 & 0.0 & 1.1 & 16.2 & 47.9 & 30.8 & 3.9 & 0.1 & 0.0 & & \\
\hline 10 & 0.0 & 0.2 & 6.4 & 34.6 & 43.8 & 14.3 & 0.7 & 0.0 & 0.0 & \\
\hline 12 & 0.0 & 0.0 & 0.7 & 9.2 & 34.7 & 40.0 & 14.0 & 1.4 & 0.0 & 0.0 \\
\hline 15 & 0.0 & 0.0 & 0.0 & 0.5 & 5.9 & 23.0 & 38.8 & 25.2 & 6.2 & 0.4 \\
\hline
\end{tabular}

\section{B. Stability of the fixed points}

After having shown the existence of the fixed points for single FD processes and extending this calculation to complete cycles, one needs to compute their stability. Since the calculation of the eigenvalues of the matrices is a lengthy but straightforward procedure we have left it for the Appendix. There we show that the results easily obtained for three and four oscillators also apply to the general case, i.e., for $\varepsilon<0$ the fixed points are attractors, whereas in the opposite case they are repellers. On the one hand, the attractiveness of the fixed points enables the formation of spatiotemporal patterns of phase-locked oscillators. This works not only for the structures with different phases but also for the periodic ones. On the other hand, when these fixed points become repellers it causes the neighboring oscillators to synchronize and from that time on they will act as a single unit; this absorption (or dimensional reduction in our language) is iterated until the whole system acts as a single unit, which completes the mechanism of the synchronization of the lattice models with very-short range interactions we have analyzed through the paper.

\section{Computer simulations}

In order to check the validity of our results we have made computer simulations on lattices of a few oscillators. In Tables I and II we represent the percentage of the structures that the system formed by $N+1$ oscillators reaches as a stationary state for two different values of $\varepsilon$ as a function of $m$,

TABLE II. The same as Table I for $\varepsilon=-0.01$.

\begin{tabular}{rrrrrrrrrr}
\hline \hline & 1 & 2 & 3 & 4 & 5 & 6 & 7 & 8 & 9 \\
\hline 3 & 51.9 & 48.1 & & & & & & & \\
4 & 17.5 & 67.3 & 15.1 & & & & & & \\
5 & 4.7 & 46.7 & 45.0 & 3.61 & & & & & \\
6 & 1.0 & 22.8 & 54.8 & 20.7 & 0.7 & & & & \\
7 & 0.2 & 8.4 & 43.0 & 40.9 & 7.4 & 0.1 & & & \\
8 & 0.0 & 2.8 & 24.3 & 48.3 & 22.5 & 2.06 & 0.0 & & \\
9 & 0.0 & 0.8 & 11.35 & 39.7 & 37.7 & 9.7 & 0.6 & 0.0 & \\
10 & 0.0 & 0.2 & 4.4 & 26.0 & 42.9 & 23.1 & 3.4 & 0.1 & 0.0 \\
\hline \hline
\end{tabular}

which stands roughly for the phase difference between neighboring oscillators times $N+1$; see Eq. (32). There are several results in these simulations that deserve further comments. For instance, we can notice that the oscillators tend to keep the maximum phase difference. The chessboardlike structure has the largest basin of attraction when the population has an even number of oscillators, whereas for an odd number of oscillators there are two peaks with the largest phase differences. However, these results depend slightly on the strength of the coupling since the maximum percentage appears for the maximum phase difference and the larger the peak the larger the phase difference. Thus we can understand the different behavior for the two different values of $\varepsilon$. Another difference concerns the reduction and, eventually, the disappearance of the basins of attraction of the fixed points that correspond to large values of $m$. This fact also affects the time the system needs to reach the stationary state; for instance, for smaller values of $\varepsilon$ not only the jumps along one cycle are smaller but there are also more attractive fixed points. On the other hand, we have corroborated that for excitatory couplings the only possible final state is synchronization, no matter how long the transient is.

\section{CONCLUSIONS}

In order to analyze the mechanisms of synchronization and the formation of spatiotemporal structures we have introduced a very simple model of pulse-coupled oscillators: a one-dimensional ring with unidirectional coupling. Despite this apparent simplicity it conserves all the features of lowdimensional systems subjected to short-range interactions which develop large-scale structures.

Although the dynamic evolution of the system involves two time scales, a slow one for the driving and a fast one for the interaction, we have constructed return maps that gives a complete information of the system. Concerning the maps, we have been able to compute exactly the fixed points of the dynamical evolution and their stability.

For a negative (inhibitory) coupling the fixed points are attractors of the dynamics. Each one of these attractors has a well-defined basin of attraction, although in some cases those regions are not simply connected. Since the evolution is discrete there are jumps among non-connected regions that cor- 
respond to advancements between oscillators. The advancements are only important in the transient dynamics, until the phases of the oscillators lie in the final basin of attraction of the fixed point. The volumes of the basins of attraction are different and depend on the value of the coupling, as we have checked by means of computer simulations of a few oscillators. However, the states with the maximum phase difference between neighboring oscillators seems to be the preferred ones.

On the other hand, for a positive (excitatory) coupling the fixed points are repellers of the dynamical evolution. Although in a configuration space with a multiplicity of repellers one can think that the system will jump from one region to another this is not our case. There are absorbing barriers surrounding the repellers; when the system reaches one of these barriers it means that at least two neighboring oscillators have synchronized. When this happens the set of synchronized oscillators acts as a single unit that cannot be broken. From that time on we only need to consider a reduced number of units; we call this fact dimensional reduction since the new system can be described in terms of matrices with fewer components. This process of absorption is iterated until the system reaches a completely synchronized configuration.

The present work only concerns the qualitative behavior of a population of pulse-coupled oscillators; nevertheless, a quantitative behavior about the time a given population needs to reach the stationary state, either a synchronized one or a spatiotemporal pattern, would be desirable in order to complete the description of such systems. Another interesting question is related to the stability of the different structures with respect to fluctuations.

\section{ACKNOWLEDGMENT}

This work was supported by CICyT of the Spanish Government, Grant No. PB94-0897.

\section{APPENDIX}

In this appendix we will compute the bounds of the eigenvalues of the matrices $\mathbb{M}_{n}$ defined in Eqs. (2) and (3). All the information will be extracted form the characteristic polynomial $P_{N, n}(\lambda, \varepsilon)$, which corresponds to the determinant $\left|\lambda \overrightarrow{1}-\mathbb{M}_{n}\right|$. In this case we will distinguish several situations. First of all when $N+1$ and $n$ have common factors it is easy to see in the determinant that a minor that corresponds to eigenvalues of modulus 1 always exists; anyway, as we explain in the text we do not need to care about this case.

The case $n=1$ is very simple to compute. By simple inspection of the determinant it is easy to see that

$$
P_{N, 1}(\lambda, \varepsilon)=\lambda P_{N-1,1}(\lambda, \varepsilon)+\mu
$$

and hence

$$
P_{N, 1}(\lambda, \varepsilon)=\lambda^{N}+\mu \lambda^{N-1}+\mu \lambda^{N-2}+\cdots+\mu \lambda+\mu .
$$

In order to compute the case $n>1$ it is convenient to introduce the following similarity transformation matrix

$$
\left(B_{n}\right)_{i, j}=\delta_{j, m_{n} i}
$$

where again the subscripts are understood modulus $N+1$, such that when it is applied to $M_{n}, M_{n}^{\prime}=B^{\dagger} M_{n} B$, one gets

$$
\left(M_{n}^{\prime}\right)_{i, j}=\delta_{i, j+1}-\delta_{j, N}+\varepsilon \delta_{j, m_{n}} \delta_{i, m_{n}+1} .
$$

With this procedure we have converted a, in principle, complex matrix in a much simpler one. The new matrix has -1 in the last column and 1's just below the main diagonal, unless at column $m_{n}$ and row $m_{n}+1$ where it has $1+\varepsilon$. In order to compute the eigenvalues we should notice that when $\varepsilon=0$ the characteristic polynomial is

$$
P_{N, n}(\lambda, \varepsilon=0)=\lambda^{N}+\lambda^{N-1}+\lambda^{N-2}+\cdots+\lambda+1 .
$$

Then to compute it for $\varepsilon \neq 0$ we expand the determinant around the previous case and one realizes that the columns that are at the right and the rows that are below this element will not contribute. Thus one has

$$
\begin{aligned}
P_{N, n}(\lambda, \varepsilon)= & \lambda^{N}+\lambda^{N-1}+\lambda^{N-2}+\cdots+\lambda^{m_{n}} \\
& +\mu\left(\lambda^{m_{n}-1}+\cdots+1\right) .
\end{aligned}
$$

The last expression can be taken as general, i.e., including the $n=1$ case, bearing in mind that $m_{1}=N$.

It is obvious that to compute the roots of Eq. (A3) is an unnecessary task, since the only needed information concerning the stability is the bounds of the eigenvalues. In order to compute these bounds we will look at the properties of the characteristic polynomial. It can be rewritten as

$$
P_{N, n}(\lambda, \varepsilon)=\sum_{k=0}^{N} \lambda^{k}+\varepsilon \sum_{k=0}^{m_{n}-1} \lambda^{k}=\frac{\lambda^{N+1}-1}{\lambda-1}+\varepsilon \frac{\lambda^{m_{n}}-1}{\lambda-1} .
$$

Then the eigenvalues of the matrix will correspond to the roots of

$$
\left(\lambda^{N+1}-1\right)+\varepsilon\left(\lambda^{m_{n}}-1\right)=0
$$

unless the artificially introduced $\lambda=1$. These roots will verify

$$
\left|\lambda^{N+1}+\varepsilon \lambda^{m_{n}}\right|=1+\varepsilon .
$$

This modulus has upper and lower bounds given by the sum and the difference, i.e.,

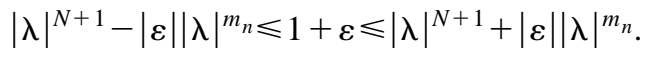

Thus for $\varepsilon>0$, we use

$$
|\lambda|^{N+1}+\varepsilon|\lambda|^{m_{n} \geqslant 1+\varepsilon},
$$

which implies that $|\lambda| \geqslant 1$. On the other hand, for $\varepsilon<0$ we take

$$
|\lambda|^{N+1}-|\varepsilon||\lambda|^{m_{n}} \leqslant 1-|\varepsilon|,
$$

which, in turn, implies that $|\lambda| \leqslant 1$. The final point is to show that the equality can only be fulfilled when both complex numbers have the same direction. It is easy to see that this can only happen when $N+1$ and $m_{n}$ have common factors. Since this fact is avoided in this demostration we are left with the fact that for an excitatory coupling $(\varepsilon>0)$ the eigenvalues are larger than 1 and the opposite for an inhibitory coupling $(\varepsilon<0)$, as we wanted to show. 
[1] C. Huygens, Letter to his father in Oeuvres Completes des Christian Huygens, edited by M. Nijhoff (Societe Hollandaise des Sciences, The Hague, Netherlands, 1893), Vol. 5, 243 (1665).

[2] A. T. Winfree, J. Theor. Biol. 16, 15 (1967).

[3] Y. Kuramoto, Chemical Oscillations, Waves and Turbulence (Springer-Verlag, Berlin, 1984).

[4] G. B. Ermentrout, J. Math. Biol. 25, 1 (1985).

[5] C. S. Peskin, Mathematical Aspects of Heart Physiology (Courant Institute of Mathematical Sciences, New York University, New York), p. 268.

[6] R. Mirollo and S. H. Strogatz, SIAM (Soc. Ind. Appl. Math.) J. Appl. Math. 50, 1645 (1990).

[7] Y. Kuramoto, Physica D 50, 15 (1991).

[8] L. F. Abbott and C. Van Vreeswijk, Phys. Rev. E 48, 1483 (1993).

[9] A. Treves, Network 4, 259 (1993).

[10] C. J. Pérez, A. Corral, A. Díaz-Guilera, K. Christensen, and A.
Arenas, Int. J. Mod. Phys. B 10, 1111 (1996).

[11] G. Goldsztein and S. H. Strogatz, Int. J. Bifurcation Chaos Appl. Sci. Eng. 5, 983 (1995).

[12] A. Díaz-Guilera, A. Arenas, A. Corral, and C. J. Pérez, Physica D 103, 419 (1997).

[13] A. Corral, C. J. Pérez, A. Díaz-Guilera, and A. Arenas, Phys. Rev. Lett. 75, 3697 (1995).

[14] H. Ito and L. Glass, Physica D 56, 84 (1992).

[15] M. de Sousa Vieira, A. J. Lichtenberg, and M. A. Lieberman, Int. J. Bifurcation Chaos Appl. Sci. Eng. 4, 1563 (1994).

[16] This assumption has physical sense in some situations. For instance, for the Peskin model of pacemaker cardiac cells [5] one can expand the PRC [12] in powers of the convexity of the driving, leading to a PRC with a constant term, whose only effect is to shift the threshold, plus another term which is proportional to the phase.

[17] J. Grasmann, Asymptotic Methods for Relaxation Oscillators and Applications (Springer-Verlag, New York, 1987). 